\title{
A hands-on activity to raise awareness about healthy sun exposure
}

\author{
Maria João Fonseca ${ }^{\mathrm{a}, \mathrm{b}, \mathrm{c}, *}$, Patrício Costa ${ }^{\mathrm{d}, \mathrm{e}, \mathrm{f}}$, Leonor Lencastre ${ }^{\mathrm{f}}$ and \\ Fernando Tavares ${ }^{\mathrm{a}, \mathrm{b}}$ \\ ${ }^{a}$ CIBIO - Centro de Investigação em Biodiversidade e Recursos Genéticos/InBIO \\ Laboratório Associado - Universidade do Porto, Vairão, Portugal; ${ }^{b}$ Faculdade de \\ Ciências, Departamento de Biologia, Universidade do Porto, Porto, Portugal; ' $I B M C-$ \\ Instituto de Biologia Molecular e Celular, Universidade do Porto, Porto, Portugal; ${ }^{d}$ Life \\ and Health Sciences Research Institute (ICVS), School of Health Sciences, University of \\ Minho, Braga, Portugal; 'ICVS/3B'S, PT Government Associate Laboratory, Braga, \\ Portugal; ${ }^{f}$ Faculdade de Psicologia e de Ciências da Educação, Universidade do Porto, \\ Porto, Portugal
}

Excessive exposure to solar ultraviolet (UV) radiation causes sunburn and has been consistently associated with increased susceptibility to melanoma and skin ageing. Acknowledging the need to promote healthy sun exposure habits, numerous educational initiatives have been developed to raise public awareness towards this issue. However, studies reveal that misinformed behaviours persist, particularly amongst young people, emphasising the need to broaden the range of interventions specifically targeting this population segment. The goal of this study was to develop and assess a hands-on activity addressing the lethal effect of sunlight. Using a mixed-method approach based on a case-control pre-/post-test design, the effectiveness of this activity was evaluated following its implementation in four high schools in Portugal. Data collected through questionnaires, classroom observations, analysis of activity reports and interviews with 150 12th-grade science students aged $17.10 \pm 0.67$ years old attending biology classes reveal that the activity had a positive impact on student knowledge about the effects of solar UV radiation and the importance of safe sunlight exposure. The implications of these findings for health and biology education - namely, concerning the instructional design of practical activities in this scope - are discussed.

Keywords: Sunlight; Ultraviolet radiation; Bacteria; High school; Hands-on activity

\section{Introduction}

Sunlight is essential for vital biological processes, such as vitamin D synthesis, in humans. However, solar ultraviolet (UV) radiation can be deleterious for living beings in

*Corresponding author. CIBIO - Centro de Investigação em Biodiversidade e Recursos Genéticos/InBIO Laboratório Associado - Universidade do Porto, Campus Agrário de Vairão, Rua Padre Armando Quintas, 4485-661 Vairão, Portugal. Email: mjfonseca@cibio.up.pt 


\section{M.J. Fonseca et al.}

general, and humans in particular. Overexposure to sunlight radiation causes sunburn, enhances skin ageing and increases the skin's susceptibility to melanoma (Armstrong 2004). Aggravated by risk factors such as a history of sunburn during childhood and overexposure to recreational sunlight, the prevalence of melanoma is raising worldwide (Carter and Donovan 2007).

Acknowledging that the promotion of healthy sun exposure habits is fundamental, numerous information campaigns and educational initiatives have been put forth to raise public awareness about these issues. Examples include The British Association of Dermatologists' 'Sun Awareness Campaign' (http://www.bad.org.uk/site/715/default.aspx) and Vichy and Killing Cancer's 'Killing Cancer Sun Awareness Campaign' (http://www.sunawareness.org/index.htm). Regardless of these endeavours, studies from various countries reveal that misinformed behaviours persist, and are particularly pervasive within the younger population segments (Cokkinides et al. 2006; Saridi et al. 2009). Therefore, it is necessary to extend, diversify and enhance the effectiveness of health education programmes seeking to promote young people's sun protection and safe sunbathing behaviours.

Considering that informed decision making in this regard depends on the critical appraisal of the consequences of sun exposure, the success of these initiatives depends upon promoting knowledge about the mutagenic and phototoxic effects of UV radiation. However, at-high school level, this understanding can be undermined by the abstract nature of the concepts required to examine this issue (namely radiation and mutation) and the need to shift from macro to micro levels of conceptualisation (Tibell and Rundgren 2010). Amongst its expected beneficial effects, practical work has been regarded as a vital part of teachers' interventions to link observable phenomena and the underlying explanations (Abrahams 2011; Rudduck and McIntyre 2007), as well as to motivate learners by providing a feel for the investigative nature of science (Hofstein and Lunetta 2003; Hume and Coll 2008). With this in mind, learner-centred practical-based approaches can be suitable instructional strategies to enhance young people's scientific literacy about sun exposure. In this context, a guided enquiry-based hands-on activity addressing sunlight's lethal effects was developed (Fonseca and Tavares 2011) and implemented in four high schools in Portugal (students aged 15-18 years old).

This study focuses on assessment of the effectiveness of this activity in promoting students' knowledge about the effects of solar UV radiation and improving their perceptions about rational sun exposure practices. In addition, the influence of the activity on students' interest in the issues discussed was evaluated. Accordingly, two research questions 35 were formulated:

- Are there significant changes in students' knowledge about the effects of solar UV radiation and perceptions about sun exposure following participation in this hands-on activity?

- How do students perceive their participation in the activity?

\section{Research design and methodology}

The activity was designed as a simple, informative and engaging practical experiment involving the use of basic microbiology procedures, such as streaking techniques and 
microbial culture and maintenance, to test the bactericidal effect of sunlight (Fonseca and Tavares 2011). The contents covered - namely, the notion and causes of mutation were framed within the 12th-grade biology programme (Direcção-Geral de Inovação e Desenvolvimento Curricular 2004), and bacteria were used as a model organism. Bacterial UV-induced damage and repair mechanisms have been well described (Goosen and Moolenaar 2008), and the rapid growth rate of these microorganisms allows obtaining clear visual results within a short time span (Fonseca and Tavares 2011). This, coupled with the activity's strong practical character, was expected to appeal to students, prompting their curiosity and motivating them to deepen their understanding of the processes addressed. The activity was structured into an introductory session followed by two lab classes, a post-lab discussion and one or two post-lab discussion classes. Figure 1 outlines the main tasks included in each of these sessions.

Using a mixed-method approach based on a case-control pre-/post-test design, the effectiveness of this activity was assessed following its implementation in four high schools in Porto from January to February 2011. A total of 140 students (aged $17.10 \pm$ 0.67 years; $59 \%$ females) from seven 12 th-grade biology classes and their teachers participated in the study. As depicted in Figure 2, the participants were organised into an experimental group comprising students subject to the intervention $(n=105)$ and a control group including students who were exposed to the same curriculum but who did not participate in the activity $(n=35)$. The participant schools were selected to be representative and specific training was provided to the teachers who implemented the activity.

To answer the first research question, a 16-item questionnaire assessing students' knowledge about UV radiation effects and perceptions about sun exposure was used as pre-/post-test (Table 1). Students' responses were examined and categorised according to a rubric defined based on the guidelines available in Crowe, Dirks, and Wenderoth (2008) and Weber (1990). The number of correct/incorrect notions and reasonable/unreasonable claims provided was quantified and compared, and each response was attributed a numerical score ranging from 0 to 6 , depending on the question considered (See Table 2). As for the second research question, a feedback questionnaire with open-ended items and a series of five-point Likert-type scales was used to assess the students' opinions about their experience. In addition, and to allow for triangulation and validation of the questionnaires' data (Cohen, Manion, and Morrison 2007; Oppenheim 1992; Plowright 2011), observational field notes on students' behaviour throughout the activity were collected, and student pair interviews and teacher interviews were performed. Students' activity reports were also analysed. For the purpose of this study, the focus is on the quantitative and interview data collected.

Responses were scrutinised and compared through descriptive and inferential statistical analyses performed with IBM SPSS Statistics 20. The first step was to ensure that the underlying assumption of normality was met for all variables. To test this assumption, we used the Kolmogorov-Smirnov test and the following rules of thumb: absolute skewness AQ1 and kurtosis values lower than 3.00 and 8.00, respectively (Kline 2011). One-sample $t$-tests were used to examine mean scores for five-point Likert-type items and values below or above the midpoint of the scale (test value $=3$ ) were considered indicative of negative or positive positioning, respectively. For the open-ended questions, differences in the preand post-test scoring rubric scores were compared using paired-samples $t$-tests. Differences 


\section{M.J. Fonseca et al.}

\section{Pre-lab Introduction}

Contextualization of the activity and presentation of its goals and aims

Introductory interactive lecture - whole class discussion prompted by the exploitation of a PowerPoint presentation (available from the authors upon request) addressing notions ranging from the effects of solar UV radiation, to safe sunbathing behaviours

Discussion of experimental set ups to assess the lethal effect of sunlight proposed by the students

Discussion of basic biosafety rules

Growth medium preparation, sterilization and platting (following procedures depicted in an alternative protocol available in Authors YYYY

Sterilization of lab material

Discussion of specific requirements of the organisms used in the activity
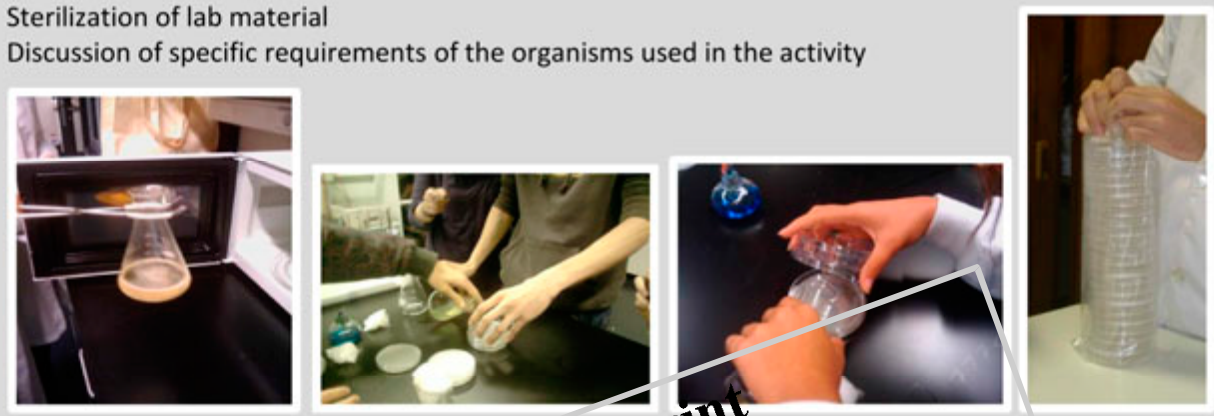

pill

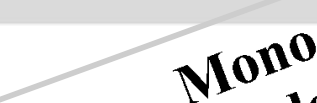

colo

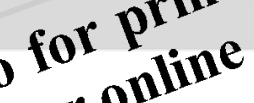
colol o

Bioassay: testing tile bactericidal effect of siilight by exposing bacterial culture plates to sunlight for increasing time periods, using differsitt filters (e.g. sunglass lenses, cardboard...)
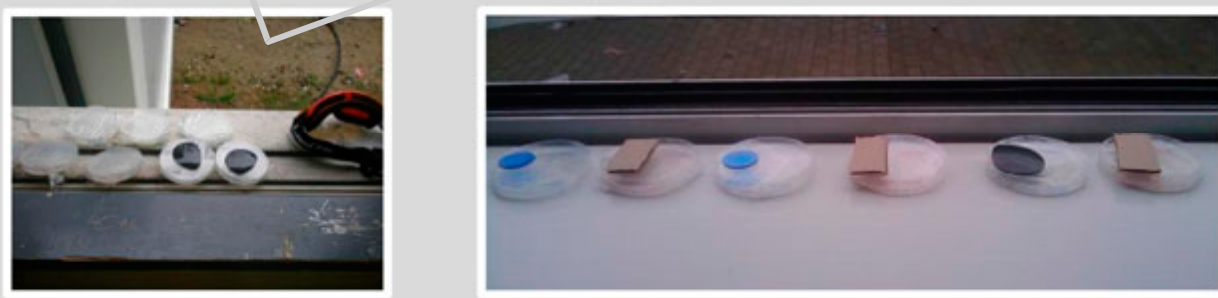

\section{Post-lab Discussion I}

Group discussion of the results obtained focusing on methodological and conceptual aspects, namely the role of the different types of filters used and debate about the effects of sunlight exposure in human cells instead of bacteria (a list of proposed discussion topics is available from the authors upon request)

Post-lab Discussion II

Classroom presentation and discussion of student activity reports

Figure 1. Outline of the main tasks performed in each of the sessions comprised in the activity. A

AQ7 detailed description of this activity is available in Authors, YYYY . All pictures were taken by the students during the lab sessions

between groups were assessed using independent-samples $t$-tests. Cohen's $d$ was used as effect size measures: $d$ values below 0.20 , between 0.21 and 0.50 , between 0.51 and 1.00 and above 1.00 were considered weak, modest, moderate and strong, respectively (Cohen, Manion, and Morrison 2007). 


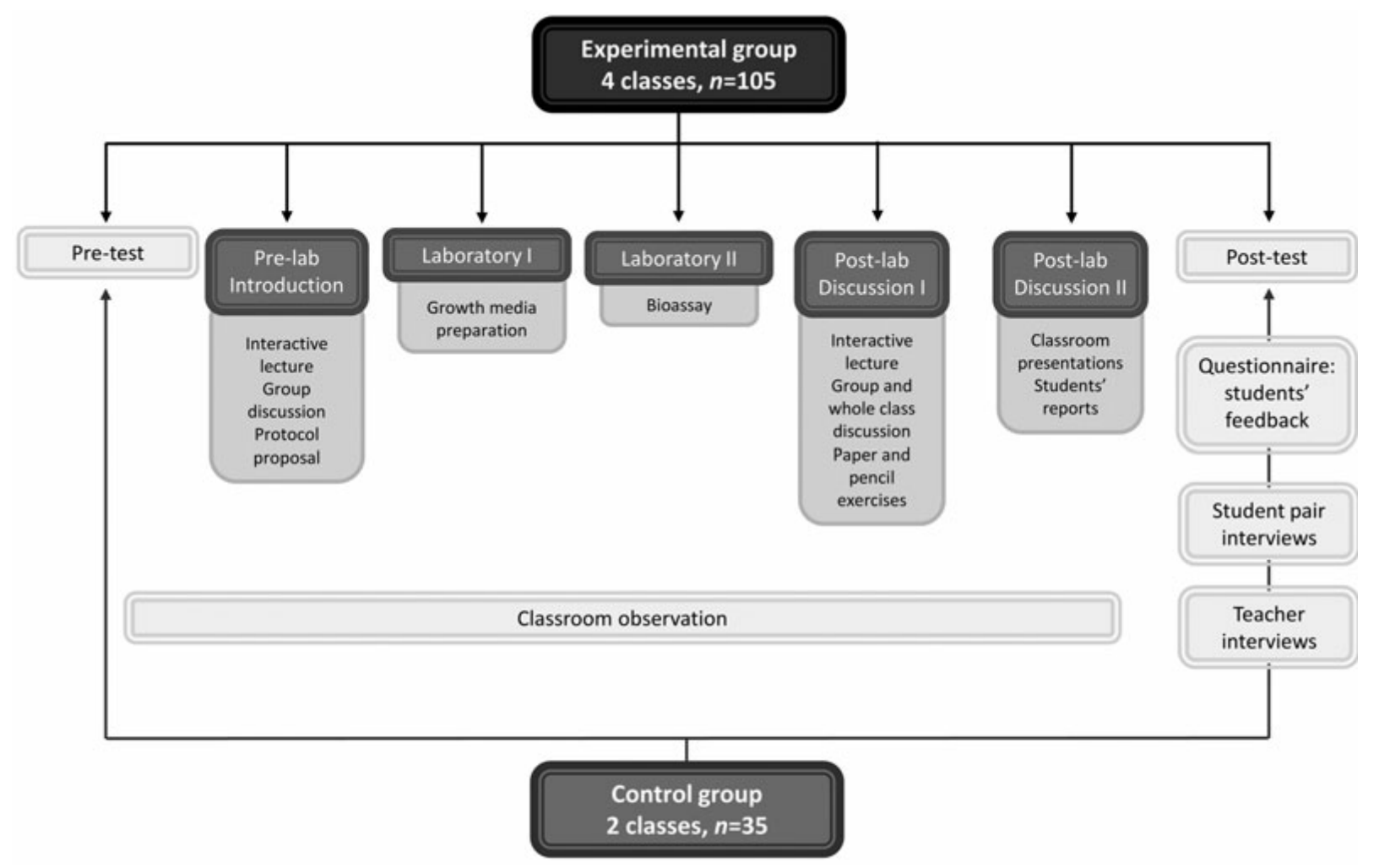

Figure 2. Activity implementation and assessment set-up

\section{Results and discussion}

\section{Students' knowledge about sunlight radiation effects}

The analysis of the experimental group students' responses indicates that there were significant pre- to post-test improvements $(p<0.05)$ for most questions pertaining to knowledge. As illustrated in Table 3, these results were consistent for both the open-ended and the true-or-false questions. For instance, following their participation in the activity, the students enhanced their knowledge about the types of electromagnetic radiation present in sunlight (Q2) and acknowledged the mutagenic effect of sunlight's UV radiation (Q5, Q6 and Q12). They also revealed a sounder understanding about the meaning of the UV index (Q8 and Q9), which can be a complex notion to grasp (Carter and Donovan 2007). Furthermore, they became more knowledgeable about the need to assure safe sun exposure habits, particularly avoiding overexposure and using protective accessories such as sunglasses (Q4, Q10 and Q11). However, the improvements observed did not enable responses within the top rubric scores levels for most questions (Table 3). In this regard, it is important to bear in mind two aspects related with the method used, which can affect the significance of the results: the difficulty of the items and the discriminating power of the scoring rubric (Goldstein and Hersen 2000; Netemeyer, Bearden, and Sharma 2003). To allow for discerning subtle variations in the quality of students' responses, a highlevel scoring rubric was developed. Nevertheless, such a degree of sophistication can result in a higher response distribution at intermediate levels than at lower and higher ones (Monette, Sullivan, and DeJong 2010). The results obtained in the control group are 
6 M.J. Fonseca et al.

Table 1. Questionnaire used as pre-/post-test

Dimension

assessed

Type of question

Question

Knowledge Open-ended

Q1. In what ways is sunlight important for life on Earth? Q2. What type(s) of electromagnetic radiation is/are present in sunlight? Q3. List some consequences of sunlight exposure for humans. Q4. In your opinion, what can be considered healthy sun exposure practices? Q5. How would you define mutation and what are its consequences? Q6. List factors that can cause mutations.

True or false with openended justification

Perceptions Likert-type scale
Q7. Our exposure to solar UV radiation can be influenced by the atmospheric pollution (T/F/DK) Q8. The UV index represents an estimate of the time required for sunburn when exposed to sunlight (T/F/DK) Q9. The UV index depends upon the temperature (T/F/DK) Q10. Once you are tanned, there is no longer need for sunscreen $(\mathrm{T} / \mathrm{F} / \mathrm{DK}) \mathrm{Q} 11$. There is no need for sunglasses when it is raining $(\mathrm{T} / \mathrm{F} / \mathrm{DK}) \mathrm{Q} 12$. Sun exposure can be associated with skin cancer (T/F/DK) Q13. There is no need to use sunscreen if I am only exposed before 11:00 and after 15:00 (F/F/DK).

Q14. Tanning through exposure to sunlight or using sunbeds makes people ( $1=$ totally disagree to $5=$ totally agree): a) look healthier; b) look older. Q15. It is adequate to expose ourselves to the sun (1-totally disagree to 5-totally agree): a) to become more attractive; b) as therapy; c) without sunscreen, if it is cloudy. Q16. I use sunscreen because (1-totally disagree to 5-totally agree): a) I want to protect my health; b) my tan is more attractive; c) doctors recommend it; d) my family asks me to.

Note: T - True; F - False; DK - 'Don't Know'

useful to clarify this aspect. Cross-comparison of the performance of the students in the two groups indicates that there were more pre- to post-test improvements within the experimental group than within the control group (Table 3). In addition, the improvements registered for the control group were not exclusive, but occurred in the experimental group as well. This suggests that the activity had a greater positive impact on students' learning than their traditional classes.

Considering that these results might have been influenced by the students' starting points (Black 1999; Oppenheim 1992), it is necessary to account for eventual differences in their knowledge prior to their participation in the activity. According to the independent-samples $t$-test results, the only statistically significant pre-test differences between groups were observed for question Q10 (Table 3), which indicates that the students in 15 both groups held identical baseline knowledge.

Regarding the differences between groups observed in the post-test, with the exception of questions Q2, Q5 and Q7, the experimental group students generally outperformed their counterparts in the control group (Table 3). While to some extent these outcomes reinforce the assumption that the learning experienced by the experimental group students 
Table 2. Example of the grading rubric used

\begin{tabular}{|c|c|c|}
\hline Question & Point & Rubric \\
\hline $\begin{array}{l}\text { Q3. List some (positive and negative) } \\
\text { consequences of sunlight exposure for humans. }\end{array}$ & $\begin{array}{l}4 \\
5 \\
1\end{array}$ & $\begin{array}{l}\text { No answer } \\
\text { No example or justification or both incorrect } \\
\text { Same amount of correct and incorrect notions } \\
\text { Only } 1 \text { correct notion } \\
2 \text { or } 3 \text { correct notions, but } 1 \text { or } 2 \text { incorrect } \\
2 \text { correct notions and none incorrect } \\
3 \text { or } 4 \text { correct notions, but } 1 \text { or } 2 \text { incorrect, } \\
\text { respectively } \\
3 \text { correct notions and none incorrect } \\
4 \text { correct notions and one incorrect } \\
\text { More than } 4 \text { correct notions, but } 2 \text { or more } \\
\text { incorrect } \\
4 \text { correct notions and none incorrect } 5 \text { or more } \\
\text { correct notions and one incorrect } \\
5 \text { or more correct notions and none incorrect } \\
\text { Only } 1 \text { reasonable notion } \\
2 \text { or } 3 \text { reasonable notions, but } 1 \text { or } 2 \\
\text { unreasonable } \\
2 \text { reasonable notions and none unreasonable } \\
3 \text { or } 4 \text { reasonable notions, but } 1 \text { or } 2 \\
\text { unreasonable, respectively } \\
3 \text { reasonable notions and none unreasonable } \\
4 \text { reasonable notions and one unreasonable } \\
\text { More than } 4 \text { reasonable notions, but } 2 \text { or more } \\
\text { unreasonable } \\
4 \text { reasonable notions and none unreasonable } \\
5 \text { or more reasonable notions and one } \\
\text { unreasonable } \\
5 \text { or more reasonable notions and none } \\
\text { unreasonable }\end{array}$ \\
\hline
\end{tabular}

Note: The full grading rubric is available from the authors upon request.

can be mainly ascribed to their engagement in the activity, it must be noted that most of the differences registered were not statistically significant. Accordingly, it can be argued that the magnitude of the improvements obtained in the experimental group did not translate into conspicuous post-test differences in relation to the control group. This raises the question of whether or not the investment required to perform this type of hands-on activity is justified by the attainable conceptual learning. The answer to this question is not straightforward as there are many aspects to account for, namely the features of the target population considered. The fact that the participants were biology students attending the last year of high school may have biased the results, as it is likely that they had previously been exposed to some of the information discussed in this activity. A way to clarify this aspect and simultaneously strengthen evidence of the effectiveness of the activity would be to extend its implementation (Lohr 2009) by targeting different age 


\begin{tabular}{|lll|}
\hline RJBE 882378 & Initial & QA: GD \\
26 February 2014 & \\
\hline
\end{tabular}

8 M.J. Fonseca et al.

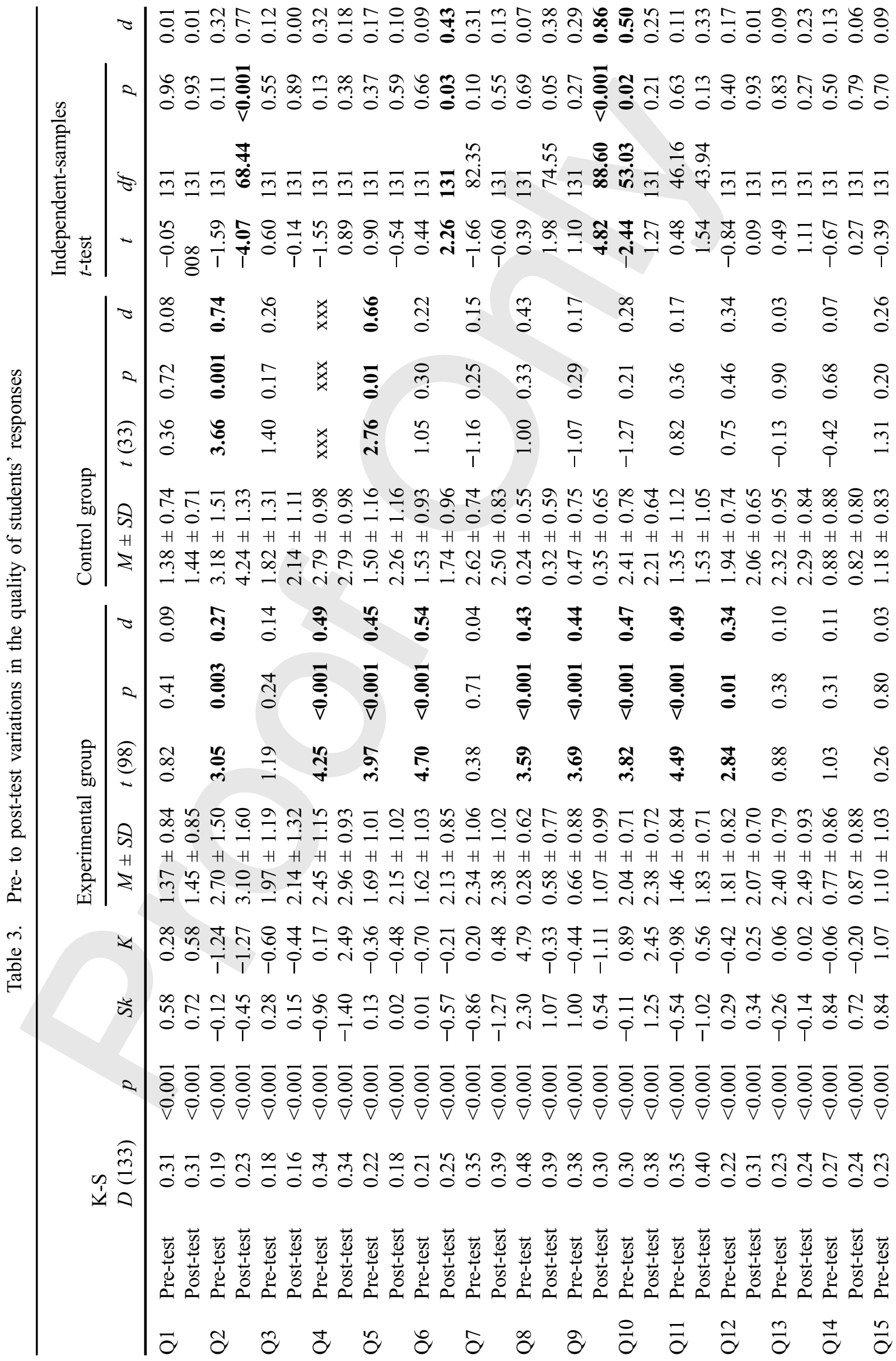




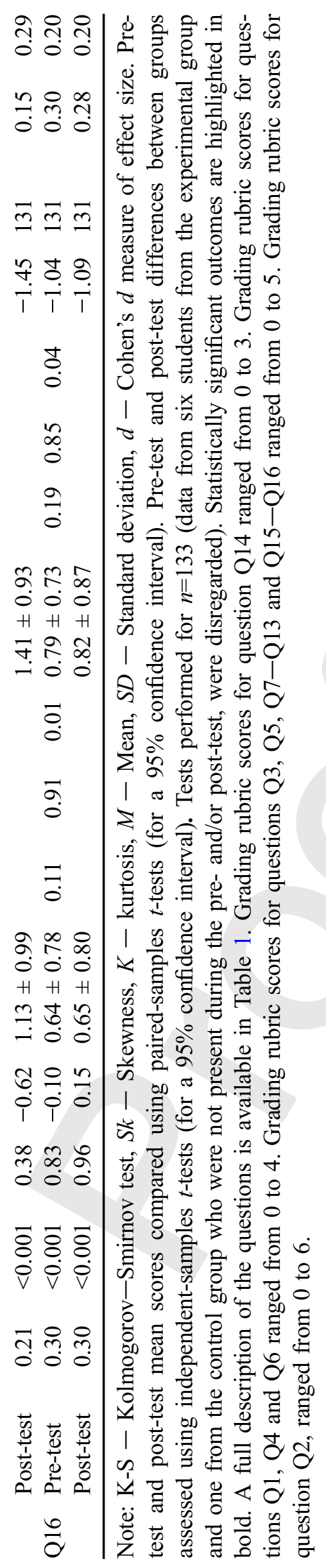




\section{M.J. Fonseca et al.}

groups, involving students from various instructional levels with diverse backgrounds and eventually considering not only formal but also informal educational contexts.

\section{Students' perceptions about sun exposure}

5 Concerning the impact of the activity on the experimental group students' perceptions, the analyses carried out revealed no major pre- to post-test variations (Figure 3). From the start, students approved sunlight exposure for therapeutic purposes and were aware that sunscreen must be used even when it is cloudy (Q15). They used sunscreen to protect their health because this was recommended by their doctors and family (Q16). It was also observed that they remained uncertain about whether or not tanning makes people look healthier, more attractive or older (Q14 and Q16). Furthermore, in addition to this lack of significant variation in the scores for the five-point Likert-type items, there were no significant improvements in the quality of the arguments used to support their claims (Table 3). The fact that the conceptual learning experienced by the students did not translate into a noticeable improvement in their perceptions is not surprising, as there are elements other than knowledge - for instance, attitudes - which modulate one's perceptions without any necessary association with conceptual determinants (Ashinoff et al. 2009; LaBat, DeLong, and Gahring 2005).

Interestingly, as evidenced in Figure 3 and Table 3, these outcomes were sustained within the control group. Furthermore, there were no major differences between groups both in the pre-test and in the post-test for the three questions considered. A closer look at the students' responses to questions Q15 and Q16 (Figure 3) shows that from the start, their answers were very close to the limits of the scale used. Consistent with what was argued for the activity's impact on participants' knowledge, it is possible that they were already aware about what can be considered healthy sun exposure practices. However, from a methodological standpoint, the chance that the items' formulation might leave room for some bias in the students' responses cannot be excluded. For instance, the questions posed might have induced the students to provide socially desired answers, regardless of whether or not they matched their own opinions and intended behaviours (Black 1999). Another possibility is that the discriminating power of items used did not allow for differentiating changes in students' perceptions, which would have required using a higher-level scale (Goldstein and Hersen 2000).

\section{Students' feedback on the activity}

In general, students were pleased with their participation in the activity (Table 4). They did not perceive the contents addressed or the techniques performed as too difficult, in spite of believing that there was some effort involved. They found the activity interesting and considered theory and practice adequately articulated. Many of them were uncertain about the usefulness of this activity for their personal lives and academic pathway. This was somewhat surprising given the opportunities that the activity provided for them to develop reasoning and procedural skills, an aspect frequently highlighted by teachers and students when justifying why they value laboratory work (Abrahams 2011). Nevertheless, students 

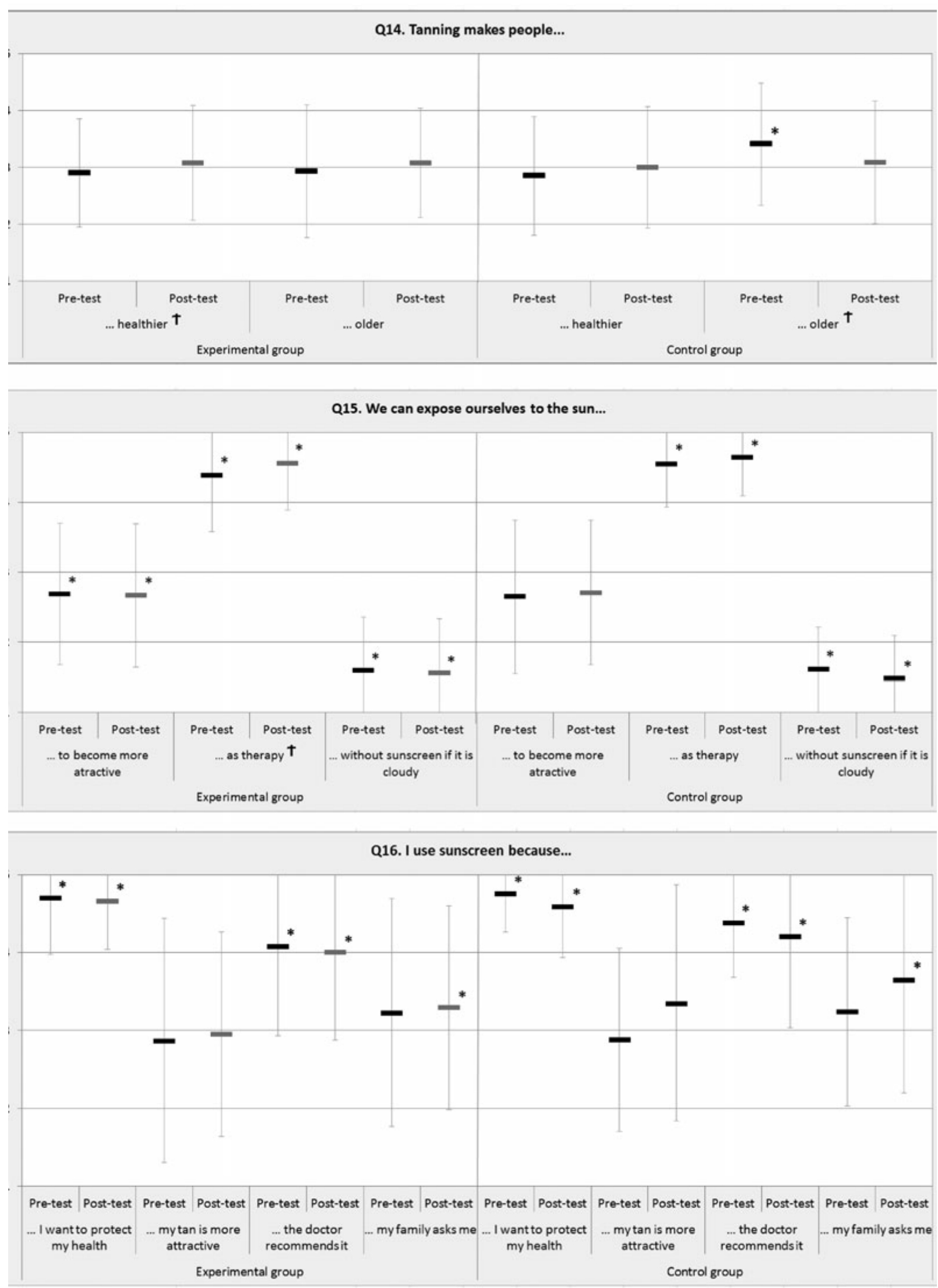

Figure 3. Students' perceptions about sun exposure 


\section{M.J. Fonseca et al.}

Table 4. Students' feedback on the activity

\begin{tabular}{lccccccc}
\hline & \multicolumn{6}{c}{ One-sample } & $\boldsymbol{t}$-test \\
\cline { 2 - 7 } & $\boldsymbol{M}$ & $\boldsymbol{S D}$ & $\boldsymbol{t}$ & $\boldsymbol{d} \boldsymbol{f}$ & $\boldsymbol{p}$ & $\boldsymbol{d}$ \\
\hline Importance of the introductory lesson & 4.00 & 0.86 & 11.76 & 103 & $<0.001$ & 1.64 \\
Contribution to understanding the issues discussed & 3.85 & 0.86 & 10.06 & 104 & $<0.001$ & 1.39 \\
Interest of the activity & 3.81 & 0.76 & 10.90 & 104 & $<0.001$ & 1.51 \\
Interest of the contents & 3.80 & 0.77 & 10.72 & 104 & $<0.001$ & 1.48 \\
Evaluation of the activity & 3.79 & 0.62 & 13.16 & 104 & $<0.001$ & 1.82 \\
Articulation between content and techniques & 3.73 & 0.75 & 9.89 & 103 & $<0.001$ & 1.37 \\
Usefulness in coping with unexpected results & 3.61 & 0.96 & 6.54 & 104 & $<0.001$ & 0.90 \\
Contribution to critical reflection about the issues discussed & 3.57 & 0.81 & 7.26 & 104 & $<0.001$ & 1.00 \\
Effort required & 3.30 & 0.68 & 4.46 & 104 & $<0.001$ & 0.63 \\
Contribution to enhanced curiosity about the issues discussed & 3.29 & 0.81 & 3.64 & 103 & $<0.001$ & 0.51 \\
Personal usefulness & 3.11 & 0.99 & 1.18 & 104 & 0.24 & 0.16 \\
Usefulness for academic pathway & 3.02 & 0.94 & 0.21 & 104 & 0.84 & 0.03 \\
Difficulty of the techniques & 2.88 & 0.72 & -1.77 & 103 & 0.08 & 0.24 \\
Difficulty of the contents & 2.63 & 0.61 & -6.14 & 102 & $<0.001$ & 0.86 \\
\hline
\end{tabular}

Note: $M-$ Mean. $S D-$ Standard deviation. $t$ - One-sample $t$-test (test value $=3$ ) for a $95 \%$ confidence interval. $d$ - Cohen's $d$ measure of effect size. Tests performed for $n=105$. Sores rated on a five-point Likert-type scale: $1=$ Very low/Not at all to $5=$ Very high/Completely. The full version of the feedback questionnaire is available from the authors upon request.

reported that the exercises helped them to better understand the issues discussed. Most importantly, they thought that the activity was helpful in assisting them to cope with unexpected results. The activity was implemented in January and February, as required to conform to the academic planning for 12th-grade biology. However, the UV index registered at this time of the year in Portugal is usually low (approximately 2-4, according to data retrieved from htup./1www.tomis.ni/f), and consequently some of the results obtained were inconclusive. Although dealing with unexpected results can be troublesome for teachers AQ2 (Kim and Tan 2010), in this study this was not regarded negatively by the students, but 10 instead encouraged them to reflect on their results and move forward. In the future, it would be interesting to change the activity's implementation schedule from winter to summer, which could be expected to allow clearer results. Another possibility would be to use black light lamps, which emit radiation in the UV-A spectrum. This could be particularly useful to call attention to the perils associated with tanning beds.

15 Also worth mentioning is the fact that the students highlighted the importance of the introductory lecture. The need to reinforce hands-on exercises with theory and guidance is a pressing issue in the current debate about the openness of enquiry-based activities (Haigh 1993; Maniotes, Caspari, and Kuhlthau 2007). The data gathered in this study support the claim that practical tasks must be explicitly contextualised within a supportAQ3 ing theoretical framework (Toplis 2012).

\section{Students and teacher interviews}

Overall, the interview data were consistent with the quantitative evidence of students' opinions. For instance, when asked which were the most appealing features of this activity, 
consistent with common reports on the contribution of practical lessons to enhance learning (Abrahams 2011; Hofstein and Lunetta 2003), most of the students claimed that they 'learn more in practical lessons than [they] do with lectures', adding that although 'you have to work more', 'it pays off'. In turn, the teachers highlighted the fact that the students were asked to propose and discuss the experimental protocol before engaging in the exercises. This aspect was purposely considered in the design of the activity, aiming to provide students with guidance and a sense of ownership that could motivate them and make them feel responsible for their own learning (Rudduck and McIntyre 2007; Toplis 2012). Although the effectiveness of open and guided enquiry-based science activities has been extensively discussed (Haigh 1993; Maniotes, Caspari, and Kuhlthau 2007), the goal herein was not to ask students to conduct an open investigation, but rather to assure a balanced level of scaffolding while at the same time avoiding recipe-like procedures. It was interesting to find that the students noticed and valued this aspect. When asked about the structure of the activity, they reported that 'proposing a protocol is hard work because [they] are not used to discuss [ing] this', but also that this 'gave [them] something to start from. It was difficult, but useful'. They also stressed the importance of the pre- and post-lab classes, stating that they 'need theory to understand what [they] are supposed to do' and 'to set up starting points' and that 'the discussion is essential to emphasise the most important aspects'.

Concerning the usefulness and interest of the activity, teachers mentioned that this sort of activity is 'essential to put in practice the theoretical concepts addressed'. They also believed that 'students like it. It gets them motivated'. These are common reasons highlighted by teachers to justify their opinions about practical work (Hofstein and Lunetta 2003). On a more practical level, teachers also mentioned that lab activities can be 'difficult to implement because [they] do not have that much time'. This calls attention to the need to provide them with time-effective procedures and proposals which are feasible in a classroom setting. As for the students, they reported that 'experiments make time pass by quicker' and that they 'like this kind of activity', which 'is useful for anyone who plans to continue studying biology'. To a certain degree, this contrasts with the feedback obtained in the questionnaire, as during the interviews students were much more certain about the usefulness of the activity for their academic pathway. It must be kept in mind that although these students were attending biology classes, many of them were not interested in continuing to study it at university.

The integration of the data gathered in the feedback questionnaire and during the interviews calls attention to four key elements which are particularly meaningful for the design of enquiry-based hands-on activities. These elements can be summarised as relevance, contextualisation, guidance and feasibility. Consistent with the recommendations found in international frameworks and syllabuses, educational activities preferably should focus on meaningful topics and contents that appeal to students' interests and are contextualised in their curricula (Bennett, Lubben, and Hogarth 2007) - an aspect that was taken under advice in this study. It is also essential to provide the participants with enough scaffolding and orientation, especially when experience and schedule limitations are significant constraints. Even if this requires compromising the openness of the enquiry-based activity, the emphasis given by the participants to the introductory theory and summing-up discussion suggests that in specific contexts, a higher degree of guidance may be a suitable option. Finally, the feasibility of the activity in actual classroom 


\section{M.J. Fonseca et al.}

settings cannot be disregarded, as it is well known that teachers have to handle practical constraints, such as time and resource limitations, while trying to meet curricular demands (Hofstein and Lunetta 2003).

\section{Conclusions and implications}

This study demonstrates that it is possible to improve high school students' knowledge about the effects of solar UV radiation and safe sun exposure practices through practical

10 work. In this specific activity, the hands-on exercises performed by the students and the visualisation of the bactericidal effects of sunlight contributed to enhance their learning about these aspects. On a general level, both students and teachers agreed upon the benefits of being given information about these issues through active learning strategies based on practical work. Furthermore, students were motivated about the contents addressed and the tasks performed, and enjoyed their experience.

The findings have implications for biology and health education practice and research. The first of these relates to the activity's instructional design. The participants' feedback suggests that students and teachers appreciate hands-on activities, which, together with the reported impact of this type of activity on students' learning, justifies their application in the context of science and health education.

Another important aspect pertains to the assessment strategy used. By setting up a case-control quasi-experimental pre-/post design following a mixed-method approach, it was possible to cross-examine and back up the interpretation of diverse types of data, allowing a more holistic depiction of the effectiveness of the intervention. Most studies assessing public perceptions and awareness about sun exposure habits have been based on large-scale surveys and have not cross-examined the respondents' self-reported views with observations of practice and tests (Ashinoff et al. 2009; Cokkinides et al. 2006; Pobbison et al. 2008). Howerer, considering cxclusively self-reported data can give a perspective of hinctoric nature, rather han reflect the respondents points of view (Cohen, Mantion, and Momison 2007 , Oppenticim 1902). Conversely, it is important to triangulate data from various sources, in order to enhance the reliability and generalisability of the findings (Plowright 2011).

In addition, it would be important to conduct a follow-up study to evaluate the effects of the activity in the longer run, particularly regarding the retention of knowledge by the students and its impact on their awareness and behaviours concerning sun exposure.

\section{Acknowledgements}

The authors are grateful to all the participant students and teachers, and to Ralph Levinson for helpful comments and suggestions on the manuscript. MJF was supported by the Fundação para a Ciência e a Tecnologia fellowship SFRH/BD/37389/2007.

\section{References}

Abrahams, I. 2011. Practical Work in Secondary Science: A Minds-on Approach. London/ New York: Continuum International Publishing Group. 
Armstrong, B. K. 2004. "How Sun Exposure Causes Skin Cancer: An Epidemiological Perspective." In Prevention of Skin Cancer, edited by D. Hill, J. M. Elwood and D. R. English, 89-116. Dordrecht: Kluwer Academic Publishers.

Ashinoff, R., V. J. Levine, A. B. Steuer, and C. Sedwick. 2009. "Teens and Tanning. Knowledge and Attitudes." Journal of Clinical and Aesthetic Dermatology 2 (2): 48-50.

Bennett, J., F. Lubben, and S. Hogarth. 2007. "Bringing Science to Life: A Synthesis of the Research Evidence on the Effects of Context-Based and STS Approaches to Science Teaching." Science Education 91 (3): 347-370.

Black, T. R. 1999. Doing Quantitative Research in the Social Sciences. an Integrated Approach to Research Design, Measurement and Statistics. London: SAGE Publications.

Carter, O. B. J., and R. J. Donovan. 2007. "Public (Mis)Understanding of the UV Index." Journal of Health Communication 12 (1): 41-52.

Cohen, L., L. Manion, and K. Morrison. 2007. Research Methods in Education. London; New York: Routledge Falmer.

Cokkinides, V., M. Weinstock, K. Glanz, J. Albano, E. Ward, and M. Thun. 2006. "Trends in Sunburns, Sun Protection Practices, and Attitudes toward Sun Exposure, Protection and Tanning among US Adolescents, 1998-2004." Pediatrics 118 (3): 853-864.

Crowe, A., C. Dirks, and M. P. Wenderoth. 2008. "Biology in Bloom: Implementing Bloom's Taxonomy to Enhance Student Learning in Biology.” CBE-Life Sciences Education 7 (4): 368-381.

Direcção-Geral de Inovação e Desenvolvimento Curricular. 2004. Programa De Biologia $12^{\circ}$ Ano. Curso Científico-Humanistico De Ciências E Tecnologias. Lisboa: Ministério da Educação.

Dobbinson, S. J., M. A. Wakefield, K. M. Jamsen, N. L. Herd, M. J. Spittal, J. E. Lipscomb, and D. J. Hill. 2008. "Weekend Sun Protection and Sunburn in Australia: Trends (1987-2002) and Association with SunSmart Television Advertising." American Journal of Preventive Medicine 34 (2): 94101.

European Space Agency. "Tropospheric Emission Monitoring Internet Service.” . Accessed September, 2013. http://www.temis.nl/

Fonseca, M. J., and F. Tavares. 2011. "The Bactericidal Effect of Sunlight." American Biology Teacher 73 (9): 548-552.

Goldstein, G., and M. Hersen. 2000. Handbook of Psychological Assessment. Oxford: Pergamon.

Goosen, N., and G. F. Moolenaar. 2008. "Repair of UV Damage in Bacteria." DNA Repair 7 (3): $353-379$.

Haigh, M. 1993. "Hands on-Minds on: Introducing Openness into Senior Biology Practical Work." Research in Science Education 23 (1): 110-117.

Hofstein, A., and V. N. Lunetta. 2003. "The Laboratory in Science Education: Foundations for the Twenty-First Century." Science Education 88 (1): 28-54.

Hume, A., and R. Coll. 2008. "Student Experiences of Carrying out a Practical Science Investigation under Direction." International Journal of Science Education 30 (9): 1201-1228.

Kim, M., and A. L. Tan. 2010. "Rethinking Difficulties of Teaching Inquiry-Based Practical Work: Stories from Elementary Pre-Service Teachers." International Journal of Science Education 33 (4): 465-486.

Kline, R. B. 2011. Principles and Practice of Structural Equation Modeling. Methodology in the Social Sciences. New York: The Guilford Press.

LaBat, Karen, Marilyn DeLong, and Sherri A. Gahring. 2005. "A Longitudinal Study of Sun-Protective Attitudes and Behaviors." Family and Consumer Sciences Research Journal 33 (3): 240-254.

Lohr, S. L. 2009. Sampling: Design and Analysis. Boston, MA: Brooks/Cole.

Maniotes, L. K., A. K. Caspari, and C. C. Kuhlthau. 2007. Guided Inquiry Learning in the 21st Century. Westport, CT: Libraries Unlimited.

Monette, D. R., T. J. Sullivan, and C. R. DeJong. 2010. Applied Social Research: A Tool for the Human Services. Belmont, CA: Cengage Learning.

Netemeyer, R. G., W. O. Bearden, and S. Sharma. 2003. Scaling Procedures. Issues and Applications. Thousand Oaks, CA: Sage Publications Inc. 
16 M.J. Fonseca et al.

Oppenheim, A. N. (1992). Questionnaire Design, Interviewing and Attitude Measurement. New ed. London; New York: Continuum.

Plowright, D. 2011. Using Mixed Methods: Frameworks for an Integrated Methodology. London: SAGE Publications.

Rudduck, J., and D. McIntyre. 2007. Improving Learning through Consulting Pupils. New York: Routledge.

Saridi, M., V. Pappa, I. Kyriazis, A. Toska, A. Giolis, A. Liachapoulou, E. Skliros, and K. Birbas. 2009. "Knowledge and Attitudes to Sun Exposure among Adolescents in Korinthos Greece." Rural and Remote Health 9 (4): 1162-1181.

The British Association of Dermatologists. Sun Awareness Campaign, last accessed September 2013, http://www.bad.org.uk/site/715/default.aspx

Tibell, L. A. E., and C.-J. Rundgren. 2010. "Educational Challenges of Molecular Life Science: Characteristics and Implications for Education and Research." CBE-Life Sciences Education 9 (1): 25-33.

Toplis, R. 2012. "Students' Views about Secondary School Science Lessons: The Role of Practical Work." Research in Science Education 42 (3): 531-549.

20 Weber, R. P. 1990. Basic Content Analysis. Newbury Park, CA: Sage Publications Inc. 